\title{
Pharmacokinetic Concentration Group Identifier
}

National Cancer Institute

\section{Source}

National Cancer Institute. Pharmacokinetic Concentration Group Identifier. NCI

Thesaurus. Code C87958.

A character or string that represents a pharmacokinetic concentration group. 